\title{
Comparison of current disruption and magnetic reconnection
}

\author{
A. T.Y. Lui*
}

\begin{abstract}
We examine similarities and differences between the concepts of current disruption (CD) and magnetic reconnection (MR). Both concepts have been invoked to account for explosive phenomena that involve energy transformation from magnetic field to charged particles. Similarities of these two concepts include (1) the occurrence of breakdown in magnetic connectivity, (2) magnetic energy as the primary energy source, and (3) plasma energization as a product of the process. Differences include (1) plasma flow across separatrix surfaces in an $X$-type magnetic field geometry being essential for MR but not for CD, (2) plasma flow ordered by the magnetic field geometry for MR but not for CD, (3) field line topology change essential for MR but not necessary for CD, and (4) CD exhibiting multifractal and symmetry breaking behavior while no such behavior has been investigated for MR. Overall, CD can be viewed as a form of generalized MR (GMR) in which the requirement for a specific magnetic field geometry and its constraint on the plasma flow pattern are removed. Therefore, the CD concept has a broader scope in applications than the MR concept alone.
\end{abstract}

\section{Background}

Identifying physical processes responsible for impulsive release of energy in the magnetosphere, and more generally in space plasmas, is an outstanding unsolved problem. These energetic processes call for the breakdown of the frozen-in-field condition such that magnetic energy can be transformed to charged particle energy. Magnetic reconnection (MR) is the most favorite mechanism invoked to account for many observed impulse energetic phenomena in the space and astrophysics communities. The concept of MR appeared first in the solar physics community, introduced by Giovanelli (1946) in an attempt to explain particle acceleration in solar flares. He suggested that flare emissions could be due to electrons accelerated by inductive electric fields near neutral points in the evolving magnetic fields of sunspots. This original concept was developed further by Sweet (1958), Parker (1957), Dungey (1961) and Petschek (1964).

\section{Magnetic reconnection}

The early concept of MR was relatively simple and developed for a two-dimensional (2D) geometry under

\footnotetext{
*Correspondence: tony.lui@jhuapl.edu
}

JHU/APL, Laurel, MD 20723-6099, USA relatively steady-state condition. In this situation, MR can be defined as the process whereby plasma flows across a surface that separates regions containing topologically different magnetic field lines. Such a separatrix surface (or line in projection) exists in a 2D configuration, where different branches of magnetic field lines join in an $X$-type neutral line (point) or, more generally, in $Y$-lines (points).

Figure 1 shows the general depiction of a MR site. Under the effect of an electric field $E_{\mathrm{y}}$ pointing out of the paper, plasma from the top and bottom with oppositely directed magnetic field lines flow with the $E_{\mathrm{y}} \times B$ drift towards the neutral sheet. This region is sometimes referred to as the plasma inflow region where magnetic field lines are visualized to move with the plasma. Since charged particles of all masses and charges move with the same $E_{\mathrm{y}} \times B$ drift, the motion of the whole population can represent the motion of the magnetic field lines contained within that population. In addition, the charged particles do not gain energy from this electric field since their motion is perpendicular to it. This condition is called the frozen-in-field, introduced by Alfvén (1942) to visualize the properties of the low-frequency electromagnetic Alfvén waves resulting from the combination of the hydrodynamic equations with Maxwell equations. 


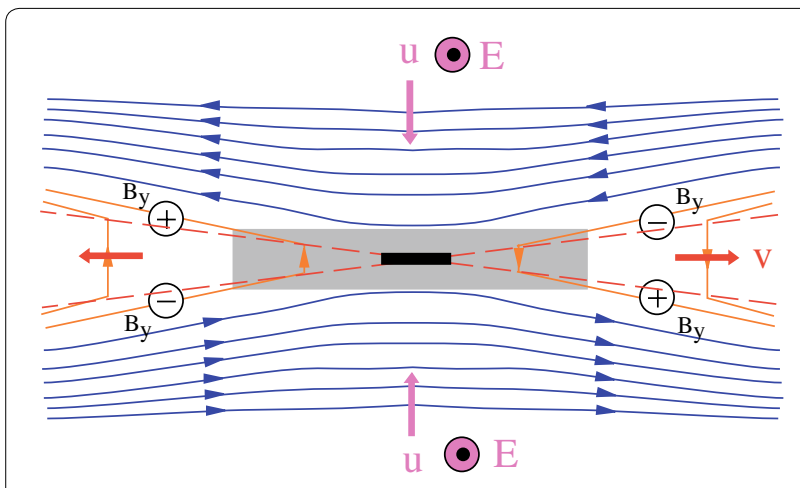

Fig. 1 A schematic diagram to illustrate the key features of a magnetic reconnection site

The central grey region is called the ion diffusion region. Inside the ion diffusion region, the motion of ions departs from that of electrons and magnetic field line motion is ill defined. This situation is often referred to as the breakdown of the frozen-in-field condition. The ions are accelerated by the Lorentz force at the separatrix surfaces, indicated by dash-lines in Fig. 1, and are ejected out on the two sides of the ion diffusion region as depicted by the red arrows in Fig. 1. The electrons still do the $E_{\mathrm{y}} \times B$ drift until they reach the electron diffusion region deep inside the ion diffusion region. The differential motion of ions and electrons in the ion diffusion region creates an electric current and an electric field $E_{\mathrm{z}}$ inside the ion diffusion region. The current and electric field generated this way are oppositely directed and form a dynamo that drives currents in the vicinity of the separatrix surfaces. This current system produces the quadruple pattern of magnetic perturbations pointing perpendicular to the $2 \mathrm{D}$ reconnection plane, as shown in Fig. 1 by the sign of $B_{\mathrm{y}}$.

Inside the electron diffusion region, electrons are no longer confined to $E_{\mathrm{y}} \times B$ drift and can move along the $X$-line. They gain energy from the electric field $E_{\mathrm{y}}$ in the process and eventually move out of this region.

The key outstanding problem in MR is to understand what physical processes are needed to break the frozenin-field condition in both the ion and electron diffusion regions.

Since nature is three-dimensional (3D), the depiction of $\mathrm{MR}$ in a $2 \mathrm{D}$ plane as indicated by Fig. 1 is deficient. In addition, impulsive energy release phenomena are highly transient and cannot be represented by a relatively steady-state situation. As a result, there were efforts to broaden the 2D nature of the MR concept. For example, Schindler et al. (1988) proposed generalized magnetic reconnection (GMR) to represent processes by which a breakdown of magnetic connection occurs due to a localized non-idealness in magnetohydrodynamics (MHD) formalism. However, this definition is deemed too broadbrush because it includes all non-MHD processes that are not intended for MR originally. As an example of this deficiency, the non-idealness caused by the presence of an electric field $E_{\|}$along the magnetic field line above an auroral arc would be considered as GMR even though no magnetic field line could be visualized to be "cut" and "joined" with another magnetic field line. More importantly, the bulk acceleration resulting in the whole plasma population jetting out of a MR site is absent in this case.

Another effort to generalize 2D picture of $M R$ to $3 \mathrm{D}$ situation was put forth by Lui et al. (2005) based on the outcome of a workshop held in Kiruna, Sweden on 2002 September $18-20$ to discuss the extension of MR to 3D and to non-steady-state condition. They proposed that MR process has the following intrinsic characteristics: (1) there is a plasma bulk flow across a boundary separating regions with topologically different magnetic field lines if projected on the plane of MR; (2) there can be an outof-the-plane magnetic field component (so-called guide field) present such that the reconnected magnetic flux tubes become twisted to form magnetic flux ropes; (3) the guide field cannot be stronger by an order of magnitude or more than the magnetic field in the plasma inflow region without the guide field component; (4) the region exhibiting non-ideal MHD condition should be localized to a scale comparable to the ion inertial length in the direction of the plasma inflow velocity.

\section{Current disruption}

It has long been surmised that the westward electrojet in the ionosphere during substorms (Akasofu 1964) is due to diversion of the cross-tail current in the magnetotail to the ionosphere during substorms (Atkinson 1967; Siscoe and Cummings 1969; Akasofu 1972). From an examination of highly fluctuating magnetic field in the midnight sector of the near-Earth region $\left(X_{\mathrm{gsm}} \approx-8.0 R_{\mathrm{E}}\right)$ during a substorm, Lui et al. (1988) adopted the term current disruption (CD) to describe the dynamic behavior and to connect that activity with the drastic reduction of local current to establish the current diversion of the cross-tail current during substorms. One such event, which was first reported by Takahashi et al. (1987), is presented in Fig. 2, showing the large amplitude variations of all magnetic field components.

The CD location was close to the Earth and the magnetic field there was strong even though its magnitude before $C D$ was much reduced from the dipole value. The magnetic field characteristics was unlike that from the approximately one-dimensional (1D) current sheet in the magnetotail. The observed features are those expected for the transition region from dipolar field geometry to tail-like geometry. 


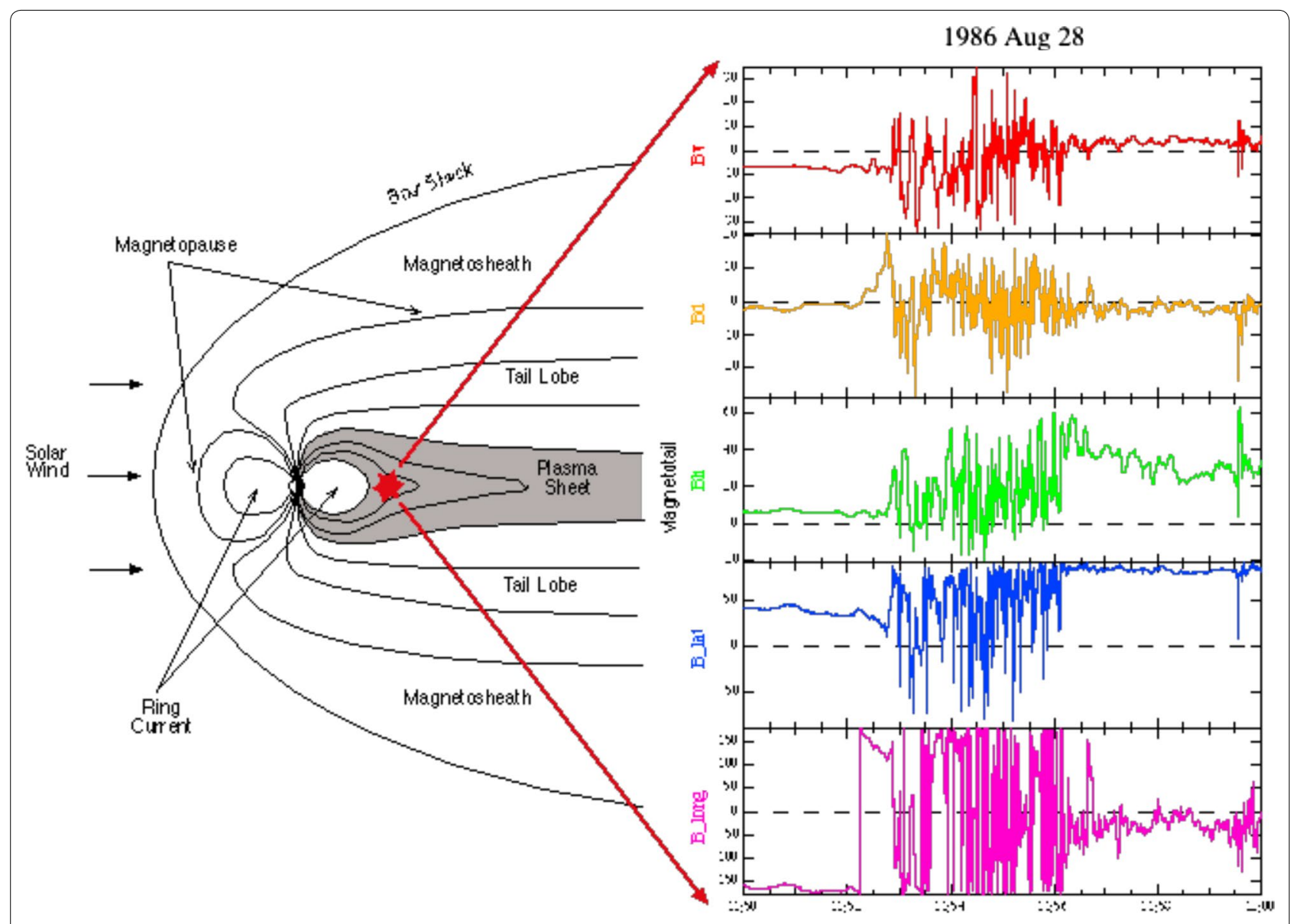

Fig. 2 Left a schematic diagram to illustrate the current disruption site observed by AMPTE/CCE satellite on 1986 August 28. Right the magnetic field components measured by the satellite for the current disruption event showing the large magnetic fluctuations at that site

Later analysis of a similar CD event showed the existence of multiple frequency components and intermittent intensification in the observed magnetic disturbances (Lui and Najmi 1997). The study indicates that CD is a multiscale process and has the intrinsic signatures of plasma turbulence. They surmised that $C D$ leads to current sheet becoming filamentary, current pattern displaying complex distribution and topology. The interval may include embedded reversals in the current density direction. Furthermore, the associated plasma flow pattern in the turbulent medium is not ordered by the magnetic field pattern, unlike that in MR.

A study of CD involves understanding plasma turbulence. Consolini and Lui $(1999,2000)$ has applied tools in complex system dynamics to examine properties of $\mathrm{CD}$. From an analysis of structure function on magnetic fluctuations to obtain the Holder exponent $H$, they noted that $H$ is about 0.5 before $C D$ onset, which is similar to Brownian motion. However, after CD, $H$ is about 0.7, implying longer persistency of magnetic fluctuations and the appearance of a new ordered phase having a different symmetry, i.e., symmetry breaking by the occurrence of $\mathrm{CD}$. In addition, they performed wavelet bicoherence analysis to show bursts of coherency indicative of intermittency in phase coupling and strong three-wave interaction. These activities can lead to inverse cascade, i.e., the decay moves from small scales to large scales, as observations indicated (Lui and Najmi 1997; Lui 2008). Further study of structure function shows the multifractal and intermittent nature of CD as well (Lui 2001). As $\mathrm{CD}$ involves plasma turbulence, it poses a challenging problem to solve since it is well recognized in the physics community at large that turbulence is an outstanding unsolved problem in classical physics (Falkovich and Sreenivasan 2006).

From the above studies, one may define $\mathrm{CD}$ as a process by which the current density reduces by a significant fraction of its value in a short time scale comparable to or shorter than the local ion gyroperiod. It has multiscale properties, characteristics of turbulence. The current reduction usually requires instability of the system. 


\section{Summary and discussion}

Energy transformation from magnetic field to charged particles is a challenging topic in space and astrophysics communities. Both MR and CD have been invoked to explain impulsive release of energy in explosive phenomena observed in nature. The above review shows some similarities and differences between the concepts of CD and MR. Similarities include (1) the occurrence of breakdown in magnetic connectivity, (2) magnetic energy as the primary energy source for the process, and (3) plasma energization as a product of the process. Differences include (1) plasma flow across separatrix surfaces in an $X$-type magnetic field geometry regarded as essential for MR but not necessarily for CD, (2) plasma flow ordered by the magnetic field geometry for MR but not for CD, (3) field line topology change essential for MR but not necessarily for $\mathrm{CD}$, and (4) CD exhibiting a multifractal and symmetry breaking behavior while no such behavior has been revealed for MR.

Overall, $C D$ is more general than $M R$ since it can release magnetic energy explosively without the requirement that magnetic topology has to be changed in the process. In this way, CD is a generalized form of MR. One advantage of adopting the CD concept is that no motion of magnetic field line is needed to describe the process. Since motion of magnetic field line is non-unique (Newcomb 1958; Vasyliunas 1972; Roth 2003), replacing MR by $C D$ can eliminate the ill-defined, and unphysical in opinions of other branches of physics, notion of magnetic field line motion. Some pioneering scientists in the space community have emphasized the inapplicability of field line reconnection in discussions of auroral and magnetospheric theory (Alfvén 1976, 1977; Parks 2004; Akasofu 2013, 2015).

\section{Acknowledgements}

This work was supported by the NSF Grants AGS-1250634 and the NASA Grants NNX12AP62G to The Johns Hopkins University Applied Physics Laboratory.

\section{Competing interests}

The author declares that he has no competing interests.

Received: 14 October 2015 Accepted: 17 November 2015

Published online: 01 December 2015

\section{References}

Akasofu S-I. The development of the auroral substorm. Planet Space Sci. 1964;12:273-82.

Akasofu SI. Magnetospheric substorms: a model. In: Dyer ER, editor. Solar terrestrial physics, Part III. D. Reidel Publ. Co. 1972. pp. 131.

Akasofu S-I. Where is the magnetic energy for the expansion phase of auroral substorms accumulated? J Geophys Res. 2013;118(1):7. doi:10.1002/201 3JA019042.
Akasofu S-I. Auroral substorms as an electrical discharge phenomenon. Prog Earth Planbetary Sci. 2015;2:20. doi:10.1186/s40645-015-0050-9.

Alfvén $\mathrm{H}$. Existence of electromagnetic-hydrodynamic waves. Nature. 1942;150:405.

Alfvén H. On frozen-in field lines and field-line reconnection. J Geophys Res. 1976;81:4019-21.

Alfvén H. Electrical currents in cosmic plasmas. Rev Geophys. 1977;15:271-84.

Atkinson G. An approximate flow equation for geomagnetic flux tubes and its application to polar substorms. J Geophys Res. 1967;72:5373-82.

Consolini G, Lui ATY. Sign-singularity analysis of current disruption. Geophys Res Lett. 1999;26:1673.

Consolini G, Lui ATY. Symmetry breaking and nonlinear wave-wave interaction in current disruption: possible evidence for a dynamical phase transition. In: Ohtani SI, Fujii R, Hesse M, Lysak RL, AGU, editors. Magnetospheric current systems. AGU geophysical monograph 118. Washington, DC. 2000. pp. 395

Dungey JW. Interplanetary magnetic field and the auroral zones. Phys Res Lett. 1961;6:47-8.

Falkovich G, Sreenivasan K. Lessons from hydrodynamic turbulence. Phys Today. 2006;59(4):43-9. doi:10.1063/1.2207037.

Giovanelli RG. A theory of chromospheric flares. Nature. 1946;158:81.

Lui ATY, Lopez RE, Krimigis SM, McEntire RW, Zanetti LJ, Potemra TA. A case study of magnetotail current sheet disruption and diversion. Geophys Res Lett. 1988;15:721-4.

Lui ATY, Najmi A-H. Time-frequency decomposition of signals in a current disruption event. Geophys Res Lett. 1997;24:3157-60.

Lui ATY. Multifractal and intermittent nature of substorm-associated magnetic turbulence in the magnetotail. J Atmos Solar Terr Phys. 2001;63:1379-85.

Lui ATY, Jacquey C, Lakhina GS, Lundin R, Nagai T, Phan T-D, Pu ZY, Roth M, Song Y, Treumann RA, Yamauchi M, Zelenyi LM. Critical issues on magnetic reconnection in space plasmas. Space Sci Rev. 2005;116:497-521.

Lui ATY, Yoon PH, Mok C, Ryu CM. Inverse cascade feature in current disruption. J Geophys Res. 2008;113:A00C06. doi:10.1029/2008JA013521.

Newcomb WA. Motion of magnetic lines of force. Ann Phys. 1958;3:347-85.

Parker EN. Sweet's mechanism for merging magnetic fields in conducting fluids. J Geophys Res. 1957;62:509-20. doi:10.1029/JZ062i004p00509.

Parks GK. Why space physics need to go beyond the MHD box. Space Sci Rev. 2004;113:97-125.

Petschek HE. Magnetic field annihilation. AAS-NASA Symposium on the Physics of Solar Flares, NASA Spec. Publ. SP-50. 1964. pp. 425-439.

Roth M. Magnetic reconnection and physical concepts, Proceedings of the Magnetic Reconnection Meeting, Kiurna, Sweden, September 2002. IRF Sci Rep. 2003;280:19-24.

Schindler K, Hesse M, Birn J. Magnetic reconnection, parallel electric field, and helicity. J Geophys Res. 1988;93:5547-57.

Siscoe GL, Cummings WD. On the cause of geomagnetic bays. Planet Space Sci. 1969;17:1795-802

Sweet, P. A. (1958), The neutral point theory of solar flares, Proceedings of the International Astronomical Union Symposium on Electromagnetic Phenomena in Cosmical Physics, No. 6 (Stockholm, 1956). pp. 123-134.

Takahashi K, Zanetti LJ, Lopez RE, McEntire RW, Potemra TA, Yumoto K. Disruption of the magnetotail current sheet observed by AMPTE/CCE. Geophys Res Lett. 1987;14:1019-22.

Vasyliunas VM. Nonuniqueness of magnetic field line motion. J Geophys Res. 1972:77:6271-4 